\title{
Current status of chimeric antigen receptor engineered $T$ cell-based and immune checkpoint blockade-based cancer immunotherapies
}

\author{
Upendra P. Hegde ${ }^{1} \cdot$ Bijay Mukherji ${ }^{1}$
}

Received: 10 August 2016 / Accepted: 22 April 2017 / Published online: 11 May 2017

(c) The Author(s) 2017. This article is an open access publication

\begin{abstract}
Adoptive cell therapies with chimeric antigen receptor (CAR) engineered $\mathrm{T}$ cells (CAR-T) and immune checkpoint inhibition (ICI)-based cancer immunotherapies have lately shown remarkable success in certain tumor types. CAR-T cell-based therapies targeting CD19 can now induce durable remissions as well as prolong disease-free survival of patients with CD19 positive treatment refractory $B$ cell malignancies and ICI-based therapies with humanized monoclonal antibodies against the $\mathrm{T}$ cell inhibitory receptors CTLA-4 and PD-1 as well as against the PD-1 ligand, PD-L1, can now achieve durable remissions as well as prolongation of life of a sizeable fraction of patients with melanoma and Hodgkin's lymphoma and non-small cell cancers. Most importantly, these immuno-therapeutic treatment modalities have raised the possibility of achieving long-term "containment" as well as "cures" for certain types of cancer. While this represents major advances in cancer immunotherapy, both modalities come with considerable toxicities, including fatalities. Although more work will be needed to bring CAR-T cell-based therapies to the bedside for most major cancers and a good deal more will be needed to make ICI-alone or in combination with other treatment modalities-work more consistently and across most major cancers, these two treatment modalities stand out as superb examples of successful translation of bench research to the bedside as well as represent real progress in the field of cancer immunotherapy.
\end{abstract}

Keywords Tumor $\cdot$ Immunotherapy $\cdot$ CAR-T cells $\cdot$ ICI

Bijay Mukherji

Mukherji@uchc.edu

1 University of Connecticut School of Medicine, 263

Farmington Avenue, Farmington, CT 06030, USA

$\begin{array}{ll}\text { Abbreviations } \\ \text { 4-1BB } & \text { TNF receptor superfamily } 9 \\ \text { ACT } & \text { Adoptive cell therapy } \\ \text { BTLA } & \text { B-and T lymphocyte attenuator } \\ \text { CAR-T cell } & \text { Chimeric antigen receptor engineered T cell } \\ \text { CR } & \text { Complete response } \\ \text { CRS } & \text { Cytokine release syndrome } \\ \text { CTLA-4 } & \text { Cytolytic T lymphocyte antigen-4 } \\ \text { ERBB2 } & \text { Epidermal growth factor receptor 2 } \\ \text { ICI } & \text { Immune checkpoint inhibition } \\ \text { IDO } & \text { Indoleamine 2,3-dioxygenase } \\ \text { LAG-3 } & \text { Lymphocyte-activation gene 3 } \\ \text { NCI } & \text { National Cancer Institute } \\ \text { OX40 } & \text { TNF receptor superfamily } 4 \\ \text { PD } & \text { Programmed death } \\ \text { PD-L } & \text { Programmed death ligand } \\ \text { PFS } & \text { Progression-free survival } \\ \text { TCR } & \text { T cell receptor } \\ \text { TIM-3 } & \text { T cell immunoglobulin and mucin-domain } \\ & \text { containing-3 } \\ \text { Tregs } & \text { Regulatory T cells }\end{array}$

\section{Introduction}

The field of cancer immunotherapy has gone through numerous peaks and valleys recording achievements and expectations as well as disappointments and disinterest. While monoclonal antibody-based therapies for cancerin one iteration or another-has a fairly long history and has shown considerable success [1], BCG has been reasonably effective in superficial bladder cancer [2], and trials of tumor infiltrating lymphocytes (TIL) in melanoma have shown some success in a fraction of patients [3], the field of cancer immunotherapy has been waiting for the infusion 
of novel ideas and real success. Lately, a series of clinical trials with fully humanized monoclonal antibodies that are capable of blocking the inhibitory signaling pathways mediated through the inhibitory $\mathrm{T}$ cell surface receptors, CTLA-4 and PD-1-usually referred to as immune checkpoint inhibition (ICI) — and immunotherapy with a patient's own $\mathrm{T}$ cells retooled to express a set of chimeric antigen receptor (CAR) exhibiting antibody specificities have shown durable complete remissions as well as prolongation of survival of patients with certain types of cancer $[4,5]$ and have emerged as remarkable success stories.

Both modalities have raised the tantalizing prospect for some cancer patients enjoying significant prolongation of life with their cancers "contained"- and if not "cured"especially for advanced melanoma [4], treatment refractory acute lymphocytic leukemia [5], and for treatment refractory Hodgkin's disease [6], and lately for non-small cell lung cancers [7]. While these results are impressive, both modalities come with substantial toxicities including fatalities at times. Fortunately, fatal side effects are relatively rare and most side effects have turned out to be "manageable". Understandably, these new immune based approaches to cancer treatment have been viewed as breakthroughs in the field and both modalities have been extensively reviewed separately $[4,5,8]$. Given that these two new modalities of tumor immunotherapy work by harnessing the formidable power of $\mathrm{T}$ cells in immune responses and have led to an unprecedented excitement in the field, an unbiased review of these two, together, would be useful. Accordingly, our purpose here is to undertake a critical examination of the reason(s) for the excitement brought on by these two new cancer treatment modalities, especially considering our long and somewhat frustrating search for effective cancer immunotherapy.

\section{The principles underlying CAR-T cell-based ACT and ICI-based tumor immunotherapies}

The therapeutic potential of adoptive transfer of a patient's own $\mathrm{T}$ cells, activated and expanded ex vivo, in cancer immunotherapy is now well established [3]. The central idea behind making CAR-T cells for tumor immunotherapy is to harness the formidable power of $\mathrm{T}$ cells in anti-tumor responses by endowing them with the power to recognize relevant tumor-associated antigens through a set of receptors bearing the structure of an antibody, free from MHC restriction, and thus to enhance the scope of $\mathrm{T}$ cell-based adoptive immunotherapy. CAR-T cells therapy, therefore, represents ACT with T cells retooled to target a tumor-associated antigen through the precision and power of an antibody. Kuwana et al. [9] first described the concept of making chimeric T cell receptors. Shortly thereafter, Eshhar's group [10] also published how to construct CAR-T cells. Given that a large number of such tumor reactive $\mathrm{T}$ cells bearing chimeric receptors could be generated from each patient, the translational potential of CAR-T cells in tumor immunothrerapy became obvious.

The fundamental principle behind ICI-based immunotherapy also aims to harness a robust $\mathrm{T}$ cell mediated immune response towards tumor-associated antigens by "taking the brake off" from the effector T cells. For, just as $\mathrm{T}$ cells are activated by signals through TCRs and costimulatory molecules, $\mathrm{T}$ cells also have receptors through which inhibitory signals can be transmitted-i.e., checkpoints or brakes. The operational principle underlying the ICI-based approach, therefore, is to unleash the host's anti-tumor effector $\mathrm{T}$ cells by blocking these checkpoints with an antagonistic antibody-i.e., inducing a robust anti'tumor T cell response by taking the "brake off". Given that the checkpoint inhibition, on its own, does not provide TCR-based signals as well as co-stimulatory signalstwo obligate necessities-for activating naïve $\mathrm{T}$ cells, it is believed that ICI primarily takes the "brake off" from antigen experienced $\mathrm{T}$ effector cells that have been rendered inactive, in vivo, by one mechanism or another as well as activates T memory cells. In this scenario, while it is widely believed that ICI does not prime naïve T cells, the precise mechanism(s) by which ICI works is not fully understood. Although it is fairly clear the major effect of the inhibitory reagents is on the effector $\mathrm{T}$ cells, given that regulatory $\mathrm{T}$ cells (Tregs) involved in the regulation of effector $\mathrm{T}$ cell activities also express inhibitory receptors, it has been suggested that anti-CTLA-4 antibodies work by potentiating $\mathrm{T}$ effector cells as well as by inhibiting Tregs [11]. This point; however, is yet to be fully settled.

\section{The development of CAR-T cell-based cancer immunotherapy}

Soon after the methodology for engrafting a chimeric antigen receptor with antibody-like specificity into $\mathrm{T}$ cells became available, Rosenberg's group at the NCI in collaboration with Eshhar at the Weizmann Institute demonstrated anti-tumor activities of adoptively transferred $\mathrm{T}$ cells made to express chimeric antibody/T cell receptors targeting folate receptors in a nude mouse model [12]. Thereafter, Rosenberg's group translated the idea into a phase I clinical trial of CAR-T cells targeting folate receptors in ovarian cancer [13]. This trial did not lead to anti-tumor effectiveness, but showed that CAR-T cells can be relatively safely administered to human patients. Thereafter, in another early clinical trial of CAR-T cells engineered to express specificity for the cell adhesion molecule, L1 (CD171), in neuroblastoma, Park et al. [14] also could not demonstrate 
objective clinical responses, but observed that the adoptively transferred cells exhibited poor persistence. Shortly thereafter, Till et al. [15] from the City of Hope and the Fred Hutchinson Cancer Research Center also reported a proof-of-concept for tumor specific chimeric $\mathrm{T}$ cell receptor-based strategy in the treatment of non-Hodgkin's lymphomas and Pule et al. [16] reported anti-tumor activities in neuroblastoma with CAR-T cell therapy targeting GD2 as a tumor-associated antigen.

Interest in tumor immunotherapy with CAR-T cells thereafter shifted to treating B cell malignancies targeting CD20/CD19. Using CD20/CD19 specific CAR-T cells, Jensen et al. [17] also found poor persistence of the CAR-T cells in patients due to anti-transgene rejection. Almost simultaneously, Kochenderfer et al. [18]; however, reported complete remission of CD19 positive advanced follicular lymphoma with the infusions of autologous CD19 CAR-T cells administered with IL-2. Interestingly, the treatment with CD19 CAR-T cells also led to the depletion of the patient's B cell lineage from blood and bone marrow [18]. Soon, several groups confirmed these observations and reported that CAR-T cell therapy targeting CD19 could achieve complete remissions, including molecular remissions, in a fair fraction of CD19 positive leukemia that were refractory to traditional systemic treatments [19-23]. Most importantly, multicenter trials confirmed these basic observations and long-term follow-ups revealed that the complete remissions could be durable [24, 25] and raised the prospect of achieving "cures" of a sizeable fraction of refractory lymphocytic leukemia patients with CD19 CAR-T cell-based therapy $[5,26]$. While this was clearly a positive development, CD19 CAR-T cell therapies came with substantial toxicities including fatalities (discussed later).

Understandably, the success with CD19 CAR-T cells in lymphocytic leukemia and lymphomas led to interests in extending this novel form of immunotherapy for other types of cancers targeting a variety of tumor-associated antigens. It also attracted substantial investments by the industries as CAR-T cells became viewed as "living drugs". Unfortunately, despite considerable efforts by the academia and the industries, an equivalent(s) of CD19 for CAR-T cell therapy in tumors other than CD19 positive leukemias is yet to emerge. This is quite intriguing and it deserves some contemplation. CAR-T cell-based therapy is after all a form of immunotherapy and the issue of antigenic specificity has been a major pillar on which the effectiveness of any form of immune therapy rests. This raises the obvious question of what is so special about CD19? CD19 is not exactly a "leukemia specific" antigen, and CD19 is not expressed on lymphocytic leukemia cells all that differentially-qualitatively or quantitatively. Normal B cells express CD19; hence CD19 CAR-T cell therapy is associated with the loss of CD19 positive normal B cells from blood and bone marrow resulting in profound hypogammaglobulinemia [5, 18, 26]. Thus, the reason how CD19 has turned out to be a good target for CAR-T cells in lymphocytic leukemia and how other antigens and epitopes exhibiting similar expression pattern on other types of tumors including other hematological malignancies are not remains unclear!

\section{Design of CAR-T cells, dose schedule, additional measures, et cetera}

A number of issues on CAR-T cell-based therapies remain unsettled. Among others, these include: (1) the ideal design of CAR-T cells (the inclusion of one or more than one co-stimulatory molecules and other molecules potentially affecting $\mathrm{T}$ cell function, survival, etc., viral vs. non-viral mechanism for gene transfer into $\mathrm{T}$ cells, ex vivo expansion methodology, et cetera); (2) the number of cells to be infused and the number of infusions for maximum benefit; (3) the need for and the nature of conditioning regimen (4), cytokine support to facilitate persistence of the infused cells, et cetera. While these issues are yet to be settled, most clinical trials have used the second generation CAR-T cells utilizing viral as well as non-viral gene transfer techniques, some form of pre-therapy lymphodepletion measure, exogenous IL-2 support or no cytokine support, and varied number of cells $\left(10^{5}-10^{9}\right.$ cells per infusion) $[5,26]$. Of note, CAR-T cell-based trials at multiple centers have tested infusions of a wide range of cell numbers and objective responses, including complete remissions, have been observed with different cell numbers [5, 26]. It is; however, by no means clear that large numbers of cells are needed to obtain complete remissions [5, 26]. In fact, complete remission has been achieved in chronic lymphocytic leukemia with as little as $1.4 \times 10^{5}$ antigen specific population split into three consecutive days [19].

\section{Adverse events with CAR-T cell therapy and risk- benefit analysis}

The side effects of CAR-T cell therapies can vary from mild constitutional syndrome (Flu-like symptoms, malaise, fever, etc.) to life-threatening situations requiring intensive care [5, 26-30]. The major toxicities usually result from: (a) inflammatory cytokines elaborated by the infused $\mathrm{T}$ cells from their cognate interactions with the target epitopes, (b) from secondary immune activation and from the activation of macrophages (usually referred to as "cytokine release syndrome or CRS" and macrophage activation syndrome), and (c) from massive target cell lysis (usually referred to as "tumor lysis syndrome") resulting in high fever, hypotension, hypoxia, intravascular coagulation, etc. [5, 26-30]. The side effects can result from the CAR-T 
cells acting against epitopes "on target" as well as "off target". At times, the toxicities can occur quickly, can lead to various organ (hepatic, gastrointestinal, respiratory, cardiovascular, endocrine, and neurological) dysfunctions, and at times they can be devastating resulting in death. Indeed, fatal pulmonary complication (such as seen with CAR-T targeting ERBB2 [31]), and a number of deaths from neurological complications-evidently from cerebral edemalately encountered in the ongoing Juno Therapeutics trial. Although the reason for the fatal neurological side effects is yet to be fully clarified, the fatalities were attributed to Fludarabine used as a part of the preparative regimen.

Understandably, CAR-T cell therapy requires careful monitoring for side effects; and depending on the nature of the side effects, adverse reactions from CAR-T cell therapies call for quick supportive cares including intensive cares, as well as additional measures when needed. Additional measures for the major toxicities include the use of systemic steroids and the anti-IL-6 receptor blocking monoclonal antibody, tocilizumab, for CRS-related toxicities [25-28].

It should be pointed out that while the various serious adverse events following CAR-T cell therapy remain a matter of concern, most of the toxicities from CD19 CAR-T cell therapy are becoming "manageable" [5, 8, 26-30]. However, as CAR-T cell therapies designed to target other antigens in other tumor types enter into the clinics, they are likely to lead to different types of "on target and off target" adverse events. Further, as different pre-conditioning regimens get introduced, they can also lead to additional toxicities and can add to the side effects from the inflammatory responses by the infused T cells. Thus', CAR-T cell therapy essentially navigates on uncharted territories. As such, a true evaluation of risk-benefit analysis of CAR-T cell therapy is presently not possible. Nonetheless, the benefits of durable complete remissions that a fair fraction of patients with B cell malignancies who have exhausted all standards of care can now enjoy with this form of therapy represent a remarkable development in cancer immunotherapy.

\section{The development of ICI-based cancer immunotherapies}

In the early days of studies of signaling pathways in $\mathrm{T}$ cells, CTLA-4 was thought to be just another co-stimulatory receptor [32]. It was; however, soon learned that the ligation of CTLA-4 with an agonist, in fact, opposes CD28driven co-stimulation and results in the down-regulation of $\mathrm{T}$ cell responses [33, 34]. As the role of CTLA-4 functioning as a brake in $\mathrm{T}$ cell responses became better understood, Allison recognized that taking the brake off from $\mathrm{T}$ cells might help unleash anti-tumor effector responses. Pursuing the idea in animal models, his group showed that the antibody mediated blockade of CTLA-4 indeed leads to tumor regression [35]. It took some time to take the idea to the bedside, but eventually the anti-CTLA- 4 antibody was humanized, industries got involved, and humanized antiCTLA-4 antibody was moved to clinical trials.

Several phase I-II trials quickly revealed anti-tumor activities of the inhibitory anti-CTLA-4 antibody [36-39] opening a novel strategy in cancer immunotherapy [40]. Interestingly, while several types of tumors showed positive response, the results of CTLA-4 blockade were most impressive in melanoma. Trials of anti-CTLA-4 blockadebased therapy continued and several multicenter-based trials of Ipilimumab, alone or in combination with gp100based immunization, confirmed anti-tumor activities of the anti-CTLA-4 antibody Ipilimumab alone, and most importantly, showed its effectiveness in improving survival of melanoma patients [41].

Yet interestingly, the best overall response rates $(95 \%$ CI) from the multicenter trials were not all that high (The response rates for Ipilimumab, gp100, and Ipilimumab + gp100 were $10.9,1.5$, and 5.7\%, respectively). No complete response was noted with gp100 alone, and the $\mathrm{CR}$ rate for Ipilimumab and Ipilimumab + gp100 were 2 and $1 \%$, respectively. However, the median survival rates were improved with Ipilimumab alone, gp100 alone, or with Ipilimumab + gp100 (The median survivals of the three groups were 10.1, 6.4 and 10 months, respectively). More importantly, the overall survivals of the three groups at 2 years (Kaplan-Meier survival curves) for the Ipilimumab, gp100, and Ipilimumab + gp100 groups were $23.5,13.7$ and $21.6 \%$, respectively [41]. Further, the disease was stabilized in a significantly larger fraction of patients receiving Ipilimumab alone or receiving Ipilimumab + gp100 and objective responses in a fraction of patients receiving re-induction therapy either with Ipilimumab alone or with Ipilimumab + gp100, were noted. The response rates of the NCI trials were not substantially different. This trial showed that Ipilimumab alone can induce complete responses in a fraction of patients and that the combinations of Ipilimumab with IL-2 or with gp100 might be little bit better [42]. Understandably, the data from the multicenter trials showing improvement in response rate and improvement in overall survival with Ipilimumab [41] generated considerable excitement in the field.

Subsequently, additional trials were launched and outcome analyses of the initial trials continued. The analyses of ten subsequent prospective trials and two retrospective studies involving little over 1800 patients revealed overall survival rates of 24 and $26 \%$ at 3 years receiving Ipilimumab + gp100 or Ipilimumab alone with a fraction of patients living at the 10 years' mark [43]. Similarly, a long-term follow-up of the NCI trial also revealed "durable" and "potentially curative tumor regressions" in melanoma with the combinations of Ipilimumab and IL-2 
or Ipilimumab and gp100 (5-year survival rates of 25 and $23 \%$, respectively, while the same with Ipilimumab alone being 13\%) [43]. Of considerable interest, some patients in the NCI trial had shown continued tumor regressions over time eventually achieving complete tumor regressions even after stoppage of the treatment. This followup also showed that although relapses in some patients were noted after 3-4 years, the survival curve flattened at 3-4 years' mark [43].

The development of ICI targeting PD-1 and PD-L1 followed a similar path. Initially in a search for the genes involved in programmed cell death, Honjo's group identified PD-1 as a programmed death associated molecule, hence the name programmed death for PD [44]. Thereafter Sharpe and Freeman, in collaboration with Honjo, discovered the natural ligands for PD-1-i.e., PD-L1 and PD-L2 - and found that the engagement of PD-1 by the ligands negatively regulate $\mathrm{T}$ cell function pretty much the same way as CTLA-4 but, in a non-overlapping manner [45]. Around the same time, Chen's group independently discovered PD-L1 that they called B7-H1 as a third co-stimulatory member of the B-7 family [46]. They went on to show that tumors cells often express the B7-H1/PD-1 ligand seemingly to escape host immune responses and recognized the potential for designing $\mathrm{T}$ cell-based cancer immunotherapy [47]. These collective observations led to the developments of reagents that could target PD-1 as well as PD-L1 as another approach to inhibitory receptor blockade-based tumor immunotherapy. In due time, phase I trials began with the anti-PD-1 antibody Nivolumab and the anti-PD-L1 antibody BMS936559. Both trials included several different types of tumors and anti-tumor activities with both reagents were shortly reported [48, 49]. Another anti-PD-1 antibody, Pembrolizumab (initially called Lambrolizumab, developed by Merck) also entered clinical trial and showed essentially identical results [50].

Interestingly, the phase I trials with Nivolumab and Lambrolizumab and with the anti-PD-L1 antibody, BMS 936559, were not mature enough for median or overall survival data, the results of the first rounds of PD-1 as well as PD-L1 targeted trials nonetheless established a number of facts. First, it was quite clear that all three reagents have anti-tumor activities (the response rates against melanoma for Nivolumab, Lambrolizumab, and BMS 936559 were 28,38 , and $17 \%$, respectively); second, Nivolumab and the anti-PD-L1 antibody exhibited varied degrees of clinical activities in tumors other than melanomas such as in lung cancer, renal cancer, and ovarian cancer); third, the reagents targeting anti-PD-1 were found to be effective in patients that have previously received Ipilimumab; and fourth, the toxicities of all three reagents seemed to be considerably less severe [48-50].
Subsequently, analyses of multiple phase III trials with anti-PD-1 antibodies involving much larger patient cohorts confirmed that not only both reagents showed anti-tumor activities, both anti-PD-1 antibodies prolonged median survivals (16.8 months for Nivolumab and 31 months for Pembrolizumab) as well as prolonged overall survival rates $(43 \%$ for Nivolumab and $49 \%$ for Pembrolizumab at 2 years) [51, 52]. Importantly, in line with the Kaplan -Meier survival curve for melanoma patients receiving Ipilimumab flattening at the 3-year mark, the survival curves with the anti-PD-1 targeted therapies were also found to flatten at the 3-year mark [51,52]. Similarly, a recent extended follow-up of the Nivolumab trial [53], presented at the American Association of Cancer Research Annual Meeting 2016 at New Orleans, showed the overall survival curve plateauing at 4 years with $34 \%$ alive at 5 years.

A head to head comparison between the CTLA-4 blocking agent, Ipilimumab, and the PD-1 blocking agent, Pembrolizumab, has been carried out [54]. When the progression-free survival (PFS) and overall survival with the two were compared, the median estimates of PFS of patients receiving Pembrolizumab10 $\mathrm{mg} / \mathrm{kg}$ every two weeks was found to be somewhat longer than that with Ipilimumab (5.5 vs. 2.8 months). The overall survival at 1 year was also significantly better with Pembrolizumab (74.1 vs. 58.2\%, respectively). Similarly, the combination of Ipilimumab and Nivolumab has also been tested [55-57]. A phase I trial including nearly 100 patients receiving the two reagents revealed a response rate of about $40 \%$ with a 2-year survival of $79 \%$. A subsequent randomized trial of the two compared with Ipilimumab alone showed a much better response rate with the combination $(61 \%$ for the combination vs. $11 \%$ for Ipilimumab alone [57]. Finally, when a combination of Ipilimumab and the standard chemotherapeutic agent for melanoma, Dacarbazine, was compared with Dacarbazine and placebo alone, a significant improvement in overall survival of previously untreated melanoma patients receiving the combination was observed [58].

\section{ICI in cancers other than melanoma}

ICI-based therapies for tumors other than melanoma have mostly been carried out with PD-1 blockade-based reagents. In fact, the very early PD-1 and PD-L1 blockadebased trials included renal cancers, non-small cell lung cancers, and ovarian cancers; and some measure of objective responses were observed in all of them [48, 49]. Shortly thereafter, objective responses with anti-PD-L1 reagent were also observed in bladder cancer [59]. However, the most impressive results with anti-PD-1/PD-L1 blockadebased treatments have been in refractory Hodgkin's disease [6], in Merkel-cell carcinoma [60], and in non-small cell 
lung cancers [7]. Importantly, PD-L1 blockade-based treatment of PD-L1 positive non-small cell cancer-a tumor that had no effective treatment-has been quite remarkable. The progression-free as well as overall survivals in patients with this disease can now be extended with anti-PD-L1 reagents [7].

\section{Relation between response rates and survival from ICI}

As pointed out earlier, the overall response rates and the complete response rates from ICI have been no better than 50 and $10 \%$, respectively. Yet checkpoint blocking-based therapies have prolonged the overall survival of patients with melanoma. This seems to defy a cardinal point earlier established by cancer chemotherapeutic trials that showed that unless complete remissions were achieved in a large fraction of the patients, partial responses and "disease stabilization" do not improve overall survival. Thus, given that the complete remission rates in the ICI-based trials were no better than 10-15\% and although some patients were likely to have undergone complete remission at a later date and some patients were likely to have received additional treatments for disease progression, the survival curve flattening at around 3-4 years is essentially unprecedented in the annals of treatment of metastatic melanomas, although the overall survival curve of patients treated with tumor infiltrating lymphocytes (TIL) has also been reported to flatten at around the same time [3].

\section{Adverse events with ICI and cost-benefit analysis}

The adverse effects of ICI-based treatments include consequences of inducing $\mathrm{T}$ cell mediated immune responses, in vivo, somewhat polyclonally. Given that the reagents target a molecule that is expressed by most all activated $\mathrm{T}$ cells and as tumor tissues as well as normal tissues contain some activated $\mathrm{T}$ cells as well as given that $\mathrm{T}$ cells bearing specificities for a variety of normal tissue associated antigens exist, taking the "brake off" of all T cells leads to on target as well as off target toxicities. The toxicities mostly include inflammatory side effects resulting general debilities and effects from "inflammation" on a variety of organ systems such as the gut, skin, lung, liver, endocrine organs (such as pituitary) with occasional fatality [48-52, 63]. Of note, the incidence of adverse events and their severity with anti-PD-1-blocking agents are somewhat less than those with the CTLA-4 blocking agent [54-57]. The incidence and the severity of the adverse events with the combination of the two are; however, substantially higher than those with monotherapy [56]. Fortunately, most of these side effects are manageable. The management principles include, in addition to systemic supportive care, the use of immunosuppressive agent such as steroids, and in refractory cases, anti-TNF alpha agent, infliximab, anti-thymocyte globulin, and mycophenolic acid [56].

When the collective data on adverse effects of ICI-based treatments are taken into consideration, the toxicities of ICI remain substantial. Nonetheless, the composite data from clinical trials involving thousands of melanoma patients have clearly shown significant prolongation of progressionfree median survivals as well as shown improvement in the overall survivals with a fraction of patients (around a third) remaining alive beyond 3 years. Significant benefits in patients with refractory Hodgkin's disease, advanced Merkel-cell carcinoma, and in non-small cell lung cancers have also been achieved. Thus, it would be fair to conclude that the benefits clearly outweigh risks and that ICI-based cancer immunotherapies do represent a true advancement in the field.

\section{Factors affecting clinical responses}

A great deal of effort has been mounted to figure out factors and biomarkers that would be predictive of treatment response and better outcome. Among the various factors that have been considered, given that tumor cells often express PD-L1or L2, the ligands for PD-1, the expression of PD-L1 or L2 was correlated with response and this was thought to be a predictive factor [61]. Subsequent analyses; however, failed to establish a clear relationship between PD-L1 expression by the tumors and treatment outcome. In this context, it should be pointed out that PD-1 blockade has been found to be remarkably effective in treatment of refractory Hodgkin's disease [6] and in non-small cell lung cancers [7]. Given that Reed-Sternberg cells in Hodgkin's disease often exhibit amplification of chromosome, 9p24.1 that codes for the PD-1 ligands [62] the possibility exists that in certain tumors showing amplification of 9p24.1, PD-L1 expression might be a factor for good outcome.

The other potentially useful biomarker for response was thought to be the intensity of immune activities in the tumors, in situ. More CD8+ T cell-based immune infiltrates and the pattern of infiltration within the tumor tissues-serving as footprints of host immune activities and making a tumor "hot"-better is the chance of response $[63,64]$. In addition, it has been suggested that PD-1 blockade induces responses by inhibiting adaptive resistance [64]. While, the evidence of brisk infiltration of CD8+ T cells, in general, the evidence of preferential accumulation of clonal T cells, in particular, and inhibition of "adaptive resistance" have been associated with good response, a definitive relationship between the intensity and the nature 
of the immune infiltrate and inhibition of adaptive resistance and treatment outcome is yet to be clearly established.

Another potentially useful biomarker of positive result that has gained considerable attention is the mutation load ("genetic basis") in a given tumor [65]. It has been suggested that tumors that exhibit more mutations are likely to attract more host immune response-especially to such mutated "neo-antigens"- and thus likely to respond to ICI-based treatments [66]. Again, while this has been forwarded as another determinant of positive outcome, more studies will be needed to establish it as a truly positive biomarker.

Finally, and somewhat ironically, the extent of autoimmune adverse effects was initially thought to be a marker for better response to the earlier CTLA-4 blockade-based trials [67]. Subsequent trials; however, have not validated this finding. Thus, despite substantial efforts at defining useful biomarkers for response to ICI-based treatments, it would be fair to say that nothing has so far been proven truly useful.

\section{Discussion}

CAR-T cell- and ICI-based tumor immunotherapies have changed the very tenor of the discussions (claims of advances and admissions of failures) on the "value" of cancer immunotherapy. An accomplished tumor immunologist who had "kept an open eye" and felt "years of frustrations" in the field recently told the authors of this article that "it has been a revolution". Setting this and other personal views (unpublishable personal communications) as well as the institutional approbation [68] aside, presently it will not be inappropriate to say that these two forms of tumor immunotherapies have finally proved that immune-based cancer therapies can be made to work in humans-albeit in certain tumor types and at some cost. Unfortunately, while the adverse effects can be serious-including fatality in some-the side effects mostly represent reflections of unleashing robust immune responses at the tumor sites as well as at certain types of normal tissues. Fortunately, while the adverse events can be quite serious and unpredictable, most side effects are becoming manageable.

CD19-CAR-T cell therapy has been granted orphan status by the FDA. Intense efforts are underway to extend both CAR-T cell-as well as ICI-based treatments across other tumor types and to improve outcomes when employed alone or in combination with other agents. FDA has already approved the use of combined CTLA-4 and PD-1 blockade-based therapy. Additional combination strategies such as ICI with GM-CSF, with GM-CSF producing cell-based vaccine, and with other stimulatory signals such as with $4-1 \mathrm{BB}$ or OX40, IDO inhibitors or combination of ACT and ICI are under consideration. A variety of other combinations with immune inhibition [such as with LAG-3, TIM-3, BTLA, etc.], chemotherapeutic agents and irradiation-as additive or designed to make a tumor more "antigenic", etc., are in the drawing board. Additionally, the combination of ICI with neo-epitope-based immunization to make the treatment more personalized is also actively pursued. Time will tell which of these strategies are likely to improve results. In this context and encouragingly, the effectiveness of the combination of ACT with IL-21-primed polyclonal cytolytic $\mathrm{T}$ cells and anti-CTLA-4 antibody in a melanoma patient that had failed prior treatments with ACT and anti-CTLA-4 has recently been reported [69]. Indeed, if the history behind the development of effective combination chemotherapies for cancer is any guide, the expectation for positive outcomes from combination immunotherapy for cancer would not be inappropriate.

This brings us to the interesting findings of long survival of patients who do not achieve complete remissions from anti-CTLA-4 as well as anti-PD-1 blockade-based therapies. Traditionally, the operative goal of any systemic anticancer therapy has been sustained complete remissions in a sizeable fraction so as to improve overall survival and to achieve "cure". Interestingly, given that ICI achieves complete remissions in no more than $15 \%$ of the patients yet the survival curves of patients receiving CTLA-4-as well as PD-1 blockade-based therapies flatten after 3 to 4 years for a sizeable fraction (a respectable third) of the patients, it is tempting to argue that we are seeing something unique that can be best described as "the containment of cancer". This seems to defy the lesson learned from earlier efforts at finding "curative" chemotherapy and it is somewhat analogous to what has been achieved with HIV-AIDS [70] and with chronic myeloid leukemia [71] as patients affected by these two diseases enjoy long life with treatments that does neither achieve total neutralization of the offending virions nor completely eliminate the leukemic cells. If the data with ICI-based therapies hold, this will be not only quite impressive; this could represent a new legitimate goal in the management of cancer-i.e., keeping cancers from progressing! The results of ICI-based cancer immunotherapy seem to suggest that this goal is achievable.

Meanwhile as innovative clinical trials continue, factors influencing outcome-i.e., response vs. no-response to therapies-get further analyzed, and relevant scientific issues behind CAR-T cells and ICI-based strategies undergo careful examination, we believe that it can now be said with a definite measure of confidence that the evidence for genuine progress in the field of cancer immunotherapy with CAR-T cell- and ICI-based strategies has been compelling; and that there are reasons to expect further progress with these two basic approaches in cancer immunotherapy. 
Acknowledgements Supported by a subcontract from Public Health Service Grant PO1 CA132681 (David Baltimore) and by a generous support to Upendra P. Hegde from the Richard and Jane Lublin Foundation.

\section{Compliance with ethical standards}

Conflict of interest The authors have no conflict of interest.

Open Access This article is distributed under the terms of the Creative Commons Attribution 4.0 International License (http://creativecommons.org/licenses/by/4.0/), which permits unrestricted use, distribution, and reproduction in any medium, provided you give appropriate credit to the original author(s) and the source, provide a link to the Creative Commons license, and indicate if changes were made.

\section{References}

1. Scott AM, Wolchok JD, Old LJ (2012) Antibody therapy of Cancer. Nat Rev Cancer 12:278-287

2. Herr HW, Morales A (2008) History of bacillus CalmetteGuerin and bladder cancer: an immunotherapy success story. J Urol 179(1):53-56

3. Rosenberg SA, Yang JC, Sherry RM et al (2011) Durable complete responses in heavily pretreated patients with metastatic melanoma using T-cell transfer immunotherapy. Clin Cancer Res 17:4550-4557

4. Sharma P, Allison JP (2015) The future of immune checkpoint therapy. Science 348:56-61

5. Gill S, June CH (2015) Going viral chimeric antigen receptor $\mathrm{T}$ cell therapy for hematological malignancies. Immunol Rev 263:68-89

6. Ansell SM, Lesokhin AM, Borrello I et al (2015) PD-1 blockade with Nivolumab in relapsed or refractory Hodgkin's lymphoma. N Engl J Med 372:311-319

7. Reck M, Rodriguez-Abreu D, Robinson AG et al (2016) Pembrolizumab versus chemotherapy for PD-L1-positive nonsmall-cell cancer. N Engl J Med 375:1823-1833

8. Holzinger A, Barden M, Abken H (2016) The growing world of CAR T cell trials: a systematic review. Cancer Immunol Immunother 65:1433-1450

9. Kuwana Y, Asakura Y, Utsunomiya N et al (1987) Expression of chimeric receptor composed of immunoglobulin-derived $\mathrm{V}$ regions and $\mathrm{T}$ cell receptor-derived $\mathrm{C}$ regions. Biochem Biophys Res Commun 149:960-968

10. Gross G, Waks T, Eshhar Z (1989) Expression of immunoglobulin- $T$ cell receptor chimeric molecules as functional receptors with antibody specificity. Proc Natl Acad Sci (USA) 86:10024-10028

11. Peggs KS, Quezada SA, Chambers CA et al (2009) Blockade of CTLA-4 on both effector and regulatory $\mathrm{T}$ cell compartments contributes to the antitumor activity of anti-CTLA-4 antibodies. J Exp Med 206:1717-1730

12. Hwu P, Yang JC, Cowherd J et al (1995) In vivo antitumor activity of $\mathrm{T}$ cells redirected with chimeric antibody/T-cell receptor genes. Cancer Res 55:3369-3373

13. Kershaw MH, Westwood JA, Parker LL et al (2006) A phase I study on adoptive immunotherapy using gene-modified $\mathrm{T}$ cells for cancer. Clin Cancer Res 12:6106-6115
14. Park JR, DiGuisto DL, Slovak M et al (2007) Adoptive transfer of chimeric antigen receptor redirected cytolytic $\mathrm{T}$ lymphocytes clones in patients with neuroblastoma. Mol Ther $15: 825-833$

15. Till BG, Jensen MC, Wang J et al (2008) Adoptive immunotherapy for indolent non-Hodgkin lymphoma and mantle cell lymphoma using genetically modified autologous CD20-specific T cells. Blood 112:2261-2271

16. Pule MA, Savoldo B, Myers GD et al (2008) Virus specific T cells engineered to co express tumor specific receptors: persistence and antitumor activity in individuals with neuroblastoma. Nat Med 14:1264-1270

17. Jensen MC, Popplewell L, Cooper LJ et al (2010) Antitransgene rejection responses contributed to attenuated persistence of adoptively transferred CD20/CD19-specific chimeric antigen receptor redirected T cells in humans. Biol Blood Marrow Transpl 16:1245-1256

18. Kochenderfer JN, Wilson WH, Janik JE et al (2010) Eradication of B-lineage cells and regression of lymphoma in a patient treated with autologous $\mathrm{T}$ cells genetically engineered to recognize CD19. Blood 116:4099-4102

19. Porter DL, Levine BL, Kalos $M$ et al (2011) Chimeric antigen receptor modified $\mathrm{T}$ cells in chronic lymphoid leukemia. $\mathrm{N}$ Engl J Med 365:725-733

20. Brentjens RJ, Riviere I, Park JH et al (2011) Safety and persistence of adoptively transferred autologous CD19-targeted $\mathrm{T}$ cells in patients with relapsed or chemotherapy refractory B-cell leukemias. Blood 118:4817-4828

21. Grupp SA, Kalos M, Barrett D et al (2013) Chimeric antigen receptor-modified T cells for acute lymphoid leukemia. N Engl J Med 368:1509-1518

22. Cruz CR, Micklethwaite KP, Savoldo B et al (2013) Infusion of donor derived CD19-redirected virus specific $\mathrm{T}$ cells for B-cell malignancies relapsed after allogeneic stem cell transplant: a phase I study. Blood 122:2965-2973

23. Maude SL, Teachey DT, Porter DL et al (2015) CD19-targeted chimeric antigen receptor T-cell therapy for acute lymphoblastic leukemia. Blood 125:4017-4023

24. Maude SL, Frey N, Shaw PA et al (2014) Chimeric antigen receptor $\mathrm{T}$ cells for sustained remissions in Leukemia. N Engl J Med 371:1507-1517

25. Curran KJ, Riviere I, Silverman LB et al (2015) Multi-center clinical trial of CAR T cells in pediatric/young adult patients with relapsed B-cell ALL [ASH abstract 2533]. Blood 126(23 suppl)

26. Park JH, Geyer MB, Brentjens R (2016) CD19 targeted CAR $\mathrm{T}$-cell therapeutics for Chimeric antigen receptor (CAR) $\mathrm{T}$ cell therapeutics for hematological malignancies. Interpreting clinical outcomes to date. Blood 127:3312-3320

27. Barrett DM, Teachey DT, Grupp SA (2014) Toxicity management for patients receiving novel T-cell engaging therapies. Curr Opin Pediatr 26:43-49

28. Davila MD, Rivere I, Wang X et al (2014) Efficacy and toxicity management of $19-28 \mathrm{z}$ CAR T cell therapy in B cell acute lymphoid leukemia. Sci Transl Med 6(224):224ra25

29. Brudno JN, Kochenderfer JN (2016) Toxicities of chimeric antigen receptor $\mathrm{T}$ cells: recognition and management. Blood 127:3321-3330

30. Bonifant CL, Jackson HJ, Brentjens RJ et al (2016) Toxicity and management in CAR T-cell therapy. Mol Ther Oncolytics 3:16011

31. Morgan RA, Yang JC, Kitano M et al (2010) Case report of a serious adverse event following the administration of T-cells transduced with chimeric antigen receptor recognizing ERBB2. Mol Ther 18:843-851 
32. Linsley P, Greene JL, Bradshaw J et al (1992) Co expression and functional cooperation of CTLA-4 and CD28 on activated T lymphocytes. J Exp Med 176:1595-1604

33. Walunas TL, Lenschow DJ, Bakker CY et al (1994) CTLA-4 can function as a negative regulator of $\mathrm{T}$ cell activation. Immunity $1: 405-413$

34. Krummel MF, Allison JP (1995) CD28 and CTLA-4 have opposing effects on the response of T cells to stimulation. J Exp Med 182:459-465

35. Leach DR, Krummel LF, Allison JP (1996) Enhancement of antitumor immunity by CTLA-4 blockade. Science 271:1734-1736

36. Yang JC, Hughes M, Kammula U et al (2007) Ipilimumab (antiCTLA-4 antibody) causes regression of metastatic renal cell cancer associated with enteritis and hypophysitis. J Immunother 30(8):825-830

37. Weber J, O’Day S, Urba W et al (2008) Phase 1/II study of Ipilimumab for patients with metastatic melanoma. J Clin Oncol 26:5950-5956

38. Hodi FS, Butler M, Oble DA et al (2008) Immunologic and clinical effects of antibody blockade of cytotoxic T lymphocyteassociated antigen 4 in previously vaccinated cancer patients. Proc Natl Acad Sci USA 105:3005-3010

39. Camacho LH, Antonia S, Sosman J et al (2009) Phase I/II trial of Tremelimumab in patients with metastatic melanoma. J Clin Oncol 27:1075-1081

40. Sharma P, Wagner K, Wolchok JD, Allison JP (2011) Novel cancer immunotherapy agents with survival benefit: recent successes and next steps. Nat Rev Cancer 11:805-812

41. Hodi FS, O’Day SJ, McDermott DF et al (2010) Improved survival with Ipilimumab in patients with metastatic melanoma. $\mathrm{N}$ Engl J Med 363:711-723

42. Prieto PA, Yang JC, Sherry RM et al (2012) CTLA-4 blockade with Ipilimumab: long-term follow-up of 177 patients with metastatic melanoma. Clin Cancer Res 18:2039-2047

43. Schadendorf D, Hodi FS, Robert C et al (2015) Pooled analysis of long-term survival data from phase II and phase III trials of Ipilimumab in unresectable or metastatic melanoma. J Clin Oncol 33:1889-1894

44. Ishida Y, Agata Y, Shibahara K, Honjo T (1992) Induced expression of PD-1, a novel member of the immunoglobulin gene superfamily, upon programmed cell death. EMBO J 11:3887-3895

45. Freeman GJ, Long AJ, Iwai Y et al (2000) Engagement of the PD-1 immunoinhibitory receptor by a novel B7 family member leads to negative regulation of lymphocyte activation. J Exp Med 192:1027-1034

46. Dong H, Gefeng Z, Tamada K et al (1999) B7-H1, a third member of the B7 family, co-stimulates $\mathrm{T}$ cell proliferation and interleukin-10 secretion. Nat Med 5:1365-1369

47. Dong H, Strome SE, Salomao DR et al (2002) Tumor associated B7-H1 promotes T-cell apoptosis: a potential mechanism of immune evasion. Nat Med 8:793-799

48. Topalian SL, Hodi FS, Brahmer JR et al (2012) Safety, activity, and immune correlates of anti-PD-1 antibody in cancer. N Engl J Med 366:2443-2454

49. Brahmer JR, Tykodi SS, Chow LQ et al (2012) Safety and activity of anti-PD-L1 antibody in patients with advanced cancer. N Engl J Med 366:2455-2465

50. Hamid O, Robert C, Daud A et al (2013) Safety and tumor responses with lambrolizumab (anti-PD-1) in melanoma. N Engl J Med 369:134-144

51. Topalian SL, Sznol M, McDermott DF et al (2014) Survival, durable tumor remission, and long-term safety in patients with advanced melanoma receiving Nivolumab. J Clin Oncol 32:1020-1030
52. Ribas A, Hamid O, Daud A et al (2016) Association of Pembrolizumab with tumor response and survival among patients with advanced melanoma. JAMA 315:1600-1609

53. Hodi FS, Klugger H, Sznol M et al (2016) Durable long-term survival in previously treated patients with advanced melanoma (MEL) who received nivolumab (NIVO) monotherapy in a phase I trial. Abstract CT001. In: Proceedings American Association Cancer Research 107th Annual Meeting 2016, April 16-20, New Orleans

54. Robert C, Schachter J, Long GV et al (2015) Pembrolizumab versus Ipilimumab in advanced melanoma. $\mathrm{N}$ Engl $\mathrm{J}$ Med 372:320-333

55. Wolchok JD, Kluger H, Callahan MK et al (2013) Nivolumab plus Ipilimumab in advanced melanoma. $\mathrm{N}$ Engl $\mathrm{J}$ Med 369:122-233

56. Postow MA, Chesney J, Pavlick AC et al (2015) Nivolumab and Ipilimumab in untreated melanoma. $\mathrm{N}$ Engl $\mathrm{J}$ Med 372:2006-2017

57. Larkin J, Chiarion-Sileni V, Gonzalez R et al (1015) Combined Nivolumab and Ipilimumab or monotherapy in untreated melanoma. N Engl J Med 373:23-34

58. Robert C, Thomas L, Bondarenko I et al (2011) Ipilimumab plus Dacarbazine for previously untreated metastatic melanoma. N Engl J Med 364:2517-2526

59. Powles T, Eder JP, Fine GD et al (2014) MPDL3280A (anti-PDL1) treatment leads to clinical activity in metastatic bladder cancer. Nature 515:558

60. Nghiem PT, Bhatia S, Lipson EJ (2016) PD-1 blockade with Pembrolizumab in advanced Merkel-cell carcinoma. N Engl J Med 374:2542-2551

61. Herbst RS, Soria JC, Kowanetz M et al (2014) Predictive correlates of response to anti-PD-L1 antibody MPDL3280A in cancer. Nature 515:563-567

62. Green MR, Monti S, Rodig S et al (2010) Integrative analysis reveals selective 9p24.1 amplification, increased PD-1 ligand expression, and further induction with JAK2 in nodular sclerosing Hodgkin lymphoma and primary mediastinal large B cell lymphoma. Blood 116:3268-3277

63. Brahmer JR, Drake CG, Wollner I et al (2010) Phase I study of single agent anti-programmed death-1 (MDX1106) in refractory solid tumors: safety, clinical activity, pharmacodynamics, and immunologic correlates. J Clin Oncol 28:3167-3175

64. Tumeh PC, Harview CL, Yearley JH et al (2014) PD-1 blockade induces responses by inhibiting adaptive resistance. Nature 515:568-571

65. Snyder A, Makarov V, Merghoub T et al (2014) Genetic basis for clinical response to CTLA-4 blockade in melanoma. N Engl J Med 371:2189-2199

66. Schumacher TN, Schreiber RD (2015) Neoantigens in cancer immunotherapy. Science 348(6230):69-74

67. Downey SG, Klapper JA, Smith FO et al (2007) Prognostic factors related to clinical response in patients with metastatic melanoma treated by CTL-associated antigen- 4 blockade. Clin Cancer Res 13:6681-6688

68. Couzin-Frankel J (2013) Breakthrough of the year 2013. Cancer immunother Sci 342:1432-1433

69. Chapuis AG, Lee SM, Thompson JA et al (2016) Combined IL-21-primed polyclonal CTL plus CTLA4 blockade controls refractory metastatic melanoma in a patient. J Exp Med 213:1133-1139

70. Chun TW, Fauci AS (2012) HIV reservoirs: pathogenesis and obstacles to viral eradication and cure. AIDS 26:1261-1268

71. Deininger M (2012) Stem cell persistence in chronic myeloid leukemia. Leuk Suppl 1(Suppl 2):546-548 